\title{
Factores relacionados con letalidad en pacientes con bacteriemia hospitalizados por patología médica en una institución de tercer nivel en Colombia, 2014-2016
}

\author{
Case fatality rate-related factors in patients with bacteremia hospitalized due medical conditions \\ in an institution of third level in Colombia, 2014-2016
} Santiago Sánchez-Pardo ${ }^{1}$, Andrés Felipe Ochoa-Díaz², Reynaldo Mauricio Rodríguez-Amaya ${ }^{1}$,
Elsa Marina Rojas-Garrido' y Alfonso J. Rodríguez-Morales ${ }^{3}$.

\begin{abstract}
${ }^{1}$ Postgrado de Medicina Interna, Hospital Universitario de Santander, Universidad Industrial de Santander, Bucaramanga. Santander, Colombia.
${ }^{2}$ Escuela de Medicina, Universidad Industrial de Santander, Bucaramanga. Santander, Colombia.

${ }^{3}$ Grupo de Investigación Salud Pública e Infección, Facultad de Ciencias de la Salud, Universidad Tecnológica de Pereira, Pereira. Risaralda, Colombia.
\end{abstract}

Financiamiento externo: ninguno, uso de recursos propios.

Los autores declaran no presentar conflictos de interés.

Recibido: 7 de octubre de 2018 / Aceptado: 14 de noviembre de 2019

\section{Resumen}

Introducción: Las infecciones del torrente sanguíneo son un problema creciente y actualmente son una amenaza para la salud pública. La bacteriemia representa aproximadamente $15 \%$ de todas las infecciones nosocomiales y afecta a $1 \%$ de los pacientes hospitalizados. Objetivo: Describir las características clínicas, epidemiológicas y microbiológicas de episodios de bacteriemia nosocomial ocurridos en un hospital colombiano. Pacientes y Métodos: Estudio retrospectivo, observacional, de corte transversal, con inclusión de pacientes adultos, hospitalizados por el Servicio de Medicina Interna en el Hospital Universitario de Santander, Bucaramanga, Colombia, durante los años 2014 a 2016. El protocolo fue aprobado por el Comité de Ética en Investigación de la Universidad Industrial de Santander. Resultados: Se revisaron 450 historias clínicas, con 148 pacientes y 182 aislados microbianos. Los antecedentes más frecuentes fueron: hipertensión arterial $(46,6 \%) \mathrm{e}$ infección por VIH (29,7\%). El sistema vascular y urinario ocuparon los sitios anatómicos más frecuentes ( 37,3 y $38,3 \%$, respectivamente). La letalidad fue de $29 \%$. Los patógenos más frecuentemente aislados fueron: Klebsiella pneumoniae, Acinetobacter baumannii, Escherichia coli, Pseudomonas aeruginosa (en suma: 49,8\%) y Staphylococcus aureus $12,1 \%$. El análisis multivariado mostró relación de la anemia con mortalidad intrahospitalaria $(\mathrm{OR}=17,3$; IC95\% 2,95-102,0). Conclusiones: La bacteriemia es una infección frecuente durante la atención hospitalaria que presenta gran mortalidad. Es destacable el predominio de aislados de enterobacterias multiresistentes. El antecedente de infección por VIH es uno de los más frecuentes el que amerita ser evaluado como grupo de riesgo.

Palabras clave: bacteriemia; nosocomial; microbiología; Enterobacteriaceae; multiresistencia; letalidad.

\begin{abstract}
Background: Bloodstream infections are an increasing problem and currently represent a threat to public health, overcoming diseases such as HIV. Bacteremia accounts for approximately $15 \%$ of all nosocomial infections and affects $1 \%$ of all hospitalized patients. Aim: To describe the clinical, epidemiological and microbiological characteristic of episodes of nosocomial bacteremia occurring in a Colombian hospital. Methods: Retrospective, observational, cross-sectional study including adult patients, hospitalized in the internal medicine unit at the University Hospital of Santander, Bucaramanga, Colombia, during years 2014 to 2016, who met the criteria of the CDC for bloodstream infection. The protocol was approved by the Hospital Ethics Committee and by the Research Ethics Committee of the Industrial University of Santander. Results: We reviewed 450 clinical records with 148 patients and 182 microbiological isolates. 53\% were male. The most frequent comorbidities were: high blood pressure $(46.6 \%)$, HIV infection (29.7\%). The vascular and urinary systems were the most frequent anatomical sites as the source of the infection (respectively $37.3 \%$ and $38.3 \%$ ). Case fatality rate was $29 \%$. The pathogens most frequently isolated were: Klebsiella pneumoniae, Acinetobacter baumannii, Escherichia coli, Pseudomonas aeruginosa (globally: $49.8 \%$ ) and Staphylococcus aureus $12.1 \%$. The multivariate analysis showed a relationship between anemia and in-hospital mortality $(\mathrm{OR}=17.3,95 \% \mathrm{CI} 2.95-102.0)$. Conclusions: Bacteremia is a frequent infection during hospital care that presents high mortality. It is noteworthy the predominance of Enterobacteriaceae isolates with broad profiles of resistance. The history of HIV infection is one of the most frequent which deserves to be evaluated as a risk group.

Keywords: bacteremia; nosocomial; microbiology; Enterobacteriaceae; multidrug resistance mortality.
\end{abstract}




\section{Introducción}

L as infecciones bacterianas del torrente sanguíneo o bacteriemias continúan siendo un importante problema de salud pública, tanto en países desarrollados como en vías de desarrollo. Se conoce que estas infecciones representan $8,7 \%$ de las infecciones adquiridas en un ambiente hospitalario ${ }^{1-10}$. Sin embargo, en muchos de países de América Latina se carece de estudios detallados al respecto, incluso existen regiones donde no hay estudio alguno publicado al respecto, como es el caso de Colombia. Para este país los datos varían dependiendo del servicio de atención, con una frecuencia que llega hasta $40 \%$ en unidades de cuidado intensivo $(\mathrm{UCI})^{10-15}$

Las infecciones del torrente sanguíneo son un problema creciente y actualmente se perfilan en el mundo como una amenaza para la salud pública, superando algunas otras enfermedades, incluso la infección por el $\mathrm{VIH}^{9-15}$

Aproximadamente 5 a $10 \%$ de los pacientes que son ingresados en hospitales en países desarrollados contraen estas infecciones, con un costo económico que supera los US\$ 7.000 millones a causa de dicha condición. Existen estudios que reportan una duplicación del costo promedio del tratamiento para pacientes en hemodiálisis con bacteriemia por $S$. aureus resistente a meticilina (SARM) $\mathrm{y}$ otros que han llegado a estimar un costo promedio de hasta US\$ 12.714 por episodio de bacteriemia por este agente ${ }^{23,24}$. El tratamiento inapropiado representa uno de los factores de riesgo más importantes para la resistencia bacteriana con la posterior generación de microorganismos multi-resistentes (en inglés multidrug resistance-MDR $)^{2-24}$.

Los microorganismos productores de $\beta$ LEE han causado brotes de infecciones MDR y altas tasas de fracaso al tratamiento empírico ${ }^{15-20}$. Escherichia coli y $K$. pneumoniae son los productores de $\beta$ LEE más comunes en el mundo y el número de aislados ha crecido en hemocultivos, sobre todo en pacientes con neoplasias hematologías y neutropenia febril ${ }^{20,21}$. Llama la atención que estos dos patógenos que inicialmente eran descritos como patógenos nosocomiales, hoy son una causa en incremento de bacteriemia de origen comunitario, así como los aislados de SARM, adquirido en la comuni$\mathrm{dad}^{20,21}$, donde este microorganismo se asocia con peores resultados clínicos en distintos grupos de pacientes ${ }^{22-26}$. Todo esto se podría deber a la falta de control en el uso de antimicrobianos en nuestra cotidianidad y la no aplicación de programas de uso racional de antimicrobianos en el ambiente hospitalario ${ }^{13,27}$.

El personal sanitario jugaría un papel relevante al transmitir estas cepas a los pacientes y la presión ecológica ejercida por los antimicrobianos de amplio espectro que se suelen administrar a pacientes críticamente enfermos, contribuiría a seleccionar estas bacterias al erradicar el resto de la microbiota normal ${ }^{28-35}$.

Se han descrito factores de riesgo para la presentación de infecciones para agentes específicos, por ejemplo bacteriemia por Pseudomonas aeuruginosa, como la sepsis grave y la neutropenia entre otros, pero los estudios de los que se dispone hasta el momento se han centrado en la resistencia antimicrobiana más que en las variables epidemiológicas más frecuentes de esta entidad ${ }^{21}$. Los estudios de los que disponemos hasta el momento tienen un carácter predominantemente microbiológico y esto sucede de igual forma con los estudios reportados para América Latina, según la literatura científica disponible ${ }^{20-37}$.

Por lo anterior, la presente investigación pretende describir cuáles son las características clínicas, epidemiológicas y microbiológicas de los pacientes hospitalizados por el Servicio de Medicina Interna del Hospital Universitario de Santander (HUS), Bucaramanga, Colombia, que presentaron infecciones del torrente sanguíneo durante los años 2014 a 2016.

\section{Pacientes y Métodos}

\section{Diseño del estudio}

Retrospectivo, observacional y analítico, de corte transversal, con selección de los pacientes con hemocultivos positivos, provenientes del Servicio de Medicina Interna. Se incluyeron hombres o mujeres sobre 14 años de edad, que cumplieran los criterios de los CDC (año 2008) para infección del torrente sanguíneo, tener un patógeno de reconocida capacidad patógena aislado en una muestra para hemocultivo -no incluye los microorganismos de la microbiota normal de la piel- como Staphylococcus aureus, Enterococcus spp, E. coli, Pseudomonas spp, Klebsiella spp, Candida spp, y otros.

Se realizó un filtro de los servicios del hospital diferentes a Medicina Interna (Ginecología, Cirugía, Ortopedia) con un total de 926 registros para 2014, 1.029 para 2015 y 1.050 para 2016. Se revisaron 450 historias clínicas con 148 pacientes finales para el análisis y 182 aislados microbiológicos, con exclusión de los pacientes de la UCI, Unidad de Hematología con enfermedad hematológica activa o necesidad de quimioterapia y aquellos con dos o más ingresos hospitalarios durante el período de observación (Figura 1).

El registro de los aislados y patrones de resistencia se lleva mediante el software WHONET que utiliza el HUS, Bucaramanga, Colombia. Las pruebas de sensibilidad a los antimicrobianos se realizaron mediante métodos estandarizados de difusión y sistemas automatizados (Phoenix $1000^{\mathrm{TM}}$ de Becton Dickinson) en concordancia con los criterios del Clinical and Laboratory Standards Institute (CLSI) los que se verificaron usando los puntos de corte 
de la versión 2007 según referencia del laboratorio clínico de la institución.

\section{Análisis estadístico}

La información fue analizada de manera descriptiva mediante el cálculo de medidas de tendencia central y de dispersión para las variables cuantitativas, y para las variables categóricas se resumieron en frecuencias absolutas y relativas y porcentajes, lo cual se muestra en el texto y tablas. Se validó el supuesto de normalidad de los datos continuos mediante la prueba de Shapiro-Wilk empleando el paquete estadístico STATA 2.0.

Se realizó inicialmente un análisis descriptivo de las variables de interés. Posteriormente se realizó un modelo de regresión logística múltiple donde las variables que alcanzaron un valor de $\mathrm{p}<0,20$ de significancia ingresaron en el modelo a través del método stepwise hacia delante y se dejaron en el modelo múltiple final sólo aquellas variables que alcanzaron valor de $\mathrm{p}<0,10$. La probabilidad de entrada fue de 0,10 y de retiro de 0,20. Los odds ratio (OR) fueron calculados con su respectivo intervalo de confianza (95\%). Se consideró un nivel de significancia con $\mathrm{p}<0,05$.

\section{Consideraciones éticas}

Este es un estudio retrospectivo que utilizó datos de las historias clínicas de pacientes. Por lo tanto, se considera "sin riesgo" de acuerdo con la clasificación establecida en la Resolución 8.430 del Ministerio de Salud de 1993, y se condujo de acuerdo con los principios declarados en la XVIII Asamblea Médica Mundial (Helsinki, 1964). El protocolo fue aprobado por el Comité de Ética Hospitalaria y el Comité de Ética en Investigación de la Universidad Industrial de Santander, Colombia.

\section{Resultados}

Se revisaron en total 450 historias clínicas con 148 pacientes y 182 aislados microbiológicos teniendo en cuenta que $16,2 \%$ presentaron infecciones polimicrobianas. El $52 \%$ correspondió al género masculino.

Los antecedentes más frecuentes fueron: hipertensión arterial $(46,6 \%)$, infección por VIH $(29,7 \%)$, diabetes mellitus $(18,2 \%)$, EPOC $(6,7 \%)$ y cirrosis $(1,3 \%)$,

La distribución de los microorganismos se muestra en la Tabla 1.

Los sitios anatómicos como fuente primaria de la infección más frecuentes fuero los sistemas urinario y vascular con 35,8 y $28,3 \%$, respectivamente; sin embargo, llama la atención el aislamiento desde piel y tejidos blandos en $29,7 \%$. Este último incluyó todas las infecciones de piel y tejidos blandos purulentas y no purulentas clasificadas por el médico clínico.

La letalidad general fue de $29 \%$, y se clasificó a la

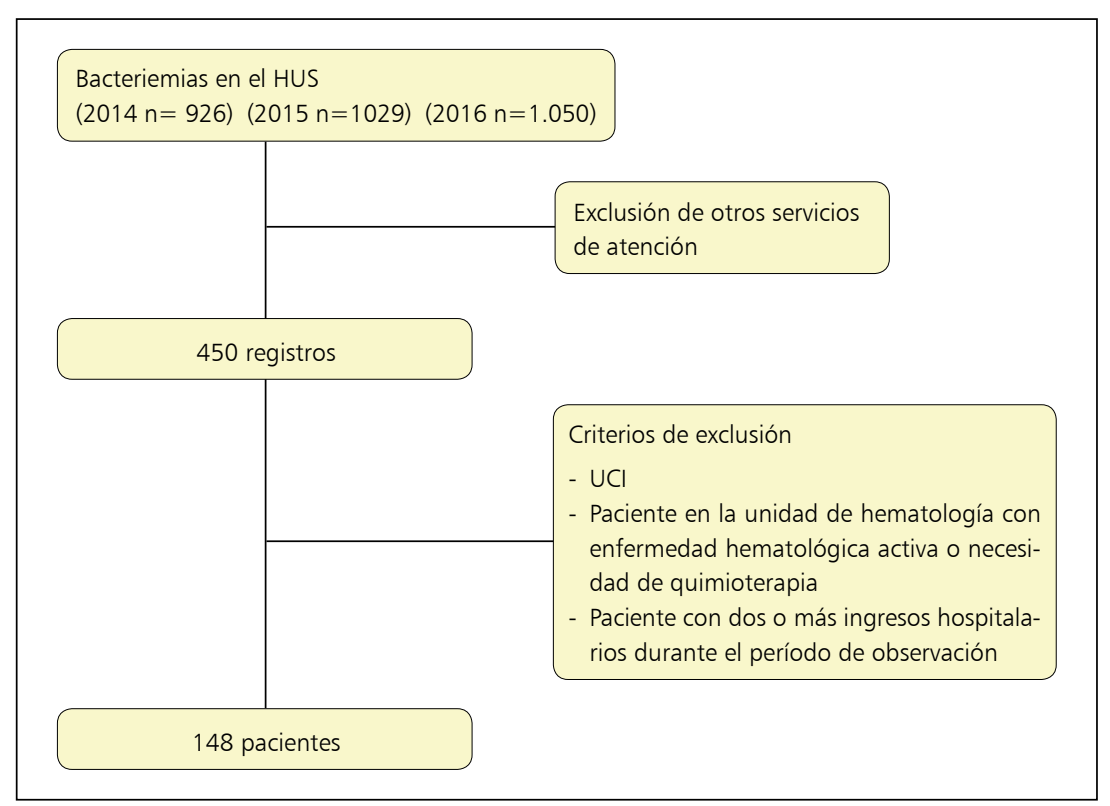

Figura 1. Algoritmo de selección de los pacientes. HUS: Hospital Universitario de Santander.

\begin{tabular}{|c|c|c|}
\hline Género y especie & $\%$ & Acumulado \\
\hline Klebsiella pneumoniae ssp & 18,9 & $61,9 \%$ \\
\hline Acinetobacter baumannii & 12,1 & \\
\hline Staphylococcus aureus & 12,1 & \\
\hline Pseudomonas aeruginosa & 10,1 & \\
\hline Escherichia coli & 8,7 & \\
\hline $\begin{array}{l}\text { Grupo SPICE (Serratia marcescens, Proteus vulgaris, Providencia spp, } \\
\text { Citrobacter freundii, Morganella morganii y Enterobacter cloacae) }\end{array}$ & & $6,0 \%$ \\
\hline \multicolumn{3}{|l|}{ Patógenos de la piel } \\
\hline Staphylococcus epidermidis & 4,0 & $13,5 \%$ \\
\hline Staphylococcus coagulasa - negativa - no identificados & 2,7 & \\
\hline Staphylococcus haemolyticus & 2,7 & \\
\hline Staphylococcus saprophyticus & 2,7 & \\
\hline Corynebacterium spp. & 0,6 & \\
\hline Staphylococcus lugdunensis & 0,6 & \\
\hline \multicolumn{3}{|l|}{ Hongos } \\
\hline Candida spp. & 1,3 & 2,7 \\
\hline Cryptococcus neoformans & 1,3 & \\
\hline Otros & & 14,8 \\
\hline
\end{tabular}

infección como intrahospitalaria $(>48 \mathrm{~h}$ posterior al ingreso hospitalario) en $86,4 \%$ de los casos; estos datos se muestran en la Tabla 2.

La mediana de días de hospitalización fue de 31 días (RIQ 19-53).

En $85,8 \%$ de los pacientes se utilizó antibioterapia empírica. 
Tabla 2. Sitios anatómicos primarios como fuente de infección y clasificación temporal de la infección

\begin{tabular}{lcc}
\hline Variable & Frecuencia & $\%$ \\
Sistema primario como fuente de infección & & \\
Sistema vascular & 42 & 28,3 \\
Sistema urinario & 53 & 35,8 \\
Sistema respiratorio & 41 & 27,7 \\
Piel y tejidos blandos & 44 & 29,7 \\
Abdominal & 35 & 23,6 \\
Clasificación temporal de la infección & & \\
Menor de 48 h & 20 & 13,5 \\
Mayor de 48 h & 128 & 86,4 \\
Antimicrobiano empírico utilizado & 127 & 85,8 \\
Desenlace clínico & & \\
Alta para domicilio & 89 & 60,1 \\
Muerte & 45 & 30,4 \\
Remisión o traslado a otra institución & 14 & 9,4 \\
\hline
\end{tabular}

\begin{tabular}{|c|c|c|}
\hline Variable & Mediana & Rango intercuartil \\
\hline Edad (años) & 55,0 & $41-70$ \\
\hline Hospitalización (días) & 31,0 & $19-53$ \\
\hline Servicio de Urgencias (días) & 3,0 & 2 a 7 \\
\hline Días de hospitalización previa & 7,0 & 4 a 17 \\
\hline Días de antimicrobiano utilizado & 7,0 & $2,7-12,2$ \\
\hline \multicolumn{3}{|l|}{ Al ingreso } \\
\hline Leucocitos (céls/ul) & 8.195 & $5.072-12.830$ \\
\hline Hemoglobina (g/dl) & 10,2 & $8,5-12,4$ \\
\hline Plaquetas (céls/ul) & 229.000 & $160.000-313.000$ \\
\hline Creatinina (mg/dl) & 0,97 & $0,69-3,34$ \\
\hline $\mathrm{NU}(\mathrm{mg} / \mathrm{dl})$ & 19,6 & $13,8-38,9$ \\
\hline ALT $(u / l)$ & 25,5 & $16,7-43$ \\
\hline AST (u/l) & 28,5 & $18,4-54,5$ \\
\hline \multicolumn{3}{|c|}{ Al momento de la obtención de los hemocultivos } \\
\hline Leucocitos (céls/ul) & 9.715 & $4.990-14.505$ \\
\hline $\mathrm{Hb}(\mathrm{g} / \mathrm{dl})$ & 9,1 & $8,1-10,7$ \\
\hline Plaquetas (céls/ul) & 201.500 & $18.250-300.000$ \\
\hline Creatinina (mg/dl) & 1,08 & $0,66-2,19$ \\
\hline $\mathrm{NU}(\mathrm{mg} / \mathrm{dl})$ & 23,6 & $13,7-43,9$ \\
\hline ALT $(u / l)$ & 27,1 & $18,5-58,5$ \\
\hline AST (u/l) & 44,0 & $24-75,3$ \\
\hline Presión arterial sistólica $(\mathrm{mmHg})$ & 120 & $106-133$ \\
\hline Presión arterial diastólica $(\mathrm{mmHg})$ & 75 & $70-80$ \\
\hline Frecuencia cardíaca (lat/min) & 93 & $79-110$ \\
\hline Temperatura $\left({ }^{\circ} \mathrm{C}\right)$ & 38,3 & $37-38,9$ \\
\hline
\end{tabular}

Las variables de laboratorio y clínicas de interés se muestran en la Tabla 3 .

Debido a que se tuvieron en cuenta los criterios del CDC de 2008 para la clasificación de la infección, se realizó el análisis de los aislados de los patógenos de la piel, ya que éstos se deben presentar en al menos dos muestras de hemocultivo con signos o síntomas de infección como fiebre (temperatura $>38^{\circ} \mathrm{C}$ ), escalofríos o hipotensión arterial. Para estos microorganismos, de 26 aislados positivos, $46 \%$ eran de dos o más muestras, $7,6 \%$ tenían hipotensión arterial y $19 \%$ fiebre.

Los antimicrobianos empíricos más frecuentemente utilizados fueron piperacilina/tazobactam, meropenem y vancomicina con 42,4-26,2 y 31,3\%, respectivamente. Los indicadores de expresión de $\beta$ LEE permiten deducir que $E$. coli y K. pneumoniae presentan un porcentaje alto de expresión de dicha enzima, dada la resistencia relativamente alta observada a las cefalosporinas de tercera y cuarta generación: cefepime (9 y 19,2\%), ceftazidima (25 y $29,6 \%$ ) y cefamicinas (cefoxitin 18,1 y $25,9 \%$ ), respectivamente.

Se realizó un análisis multivariado para letalidad letalidad incluyendo las variables clínicas y paraclínicas de importancia para este desenlace (Tabla 4). Este análisis muestra según la clasificación del índice de Pitt como grave, la leucopenia y trombocitopenia después de la obtención de los hemocultivos, anemia, hipotensión diastólica, las infecciones polimicrobianas y el número de muestras positivas de tres o más, se relacionaron con aumento de la letalidad, con diferencias estadísticamente significativas (Tabla 4).

El grupo de pacientes entre 30 y 54 años (35\%, n: 45) tuvo la mayor letalidad, lo que deja en evidencia que esta entidad causa gran letalidad y discapacidad en población de edad media.

Se realizó la clasificación del Score de mortalidad de Pitt, el cual se encarga de evaluar la gravedad, específicamente de bacteriemia, siendo categorizado como leve en $71,7 \%$, moderado $17,1 \%$ y grave $11,1 \%$.

En el grupo de pacientes con infección por VIH (n: 44), 55,8\% no tenía tratamiento anti-retroviral por ser diagnósticos nuevos y la letalidad fue de $31,8 \%$. El promedio de LTCD4+ fue de 29 céls $/ \mathrm{mm}^{3}$ y 44,1\% tenía neutropenia al momento del ingreso con recuento absoluto de neutrófilos $(\mathrm{RAN})<500$ células. Se encontró registro de carga viral en $77,2 \%$ de los pacientes (n: 34$)$, con mediana de 112.429 copias/ml (RIQ = 21.386-369.019).

Las infecciones fueron clasificadas de acuerdo al momento de la documentación microbiológica, donde la presentación mayor de $48 \mathrm{~h}$ con respecto al ingreso se observó en $93,1 \%$ de los pacientes.

El índice de gravedad de Pitt fue clasificado como leve en $68,1 \%$ y los patógenos más frecuentemente aislados fueron las enterobacterias siendo la principal $K$. 
son estudios de otros escenarios ${ }^{1,2,6,24,33,37}$ y se ratifica que la presencia de bacteriemia confiere peor desenlace a los pacientes hospitalizados con patologías médicas. Esto se asemeja a pacientes hospitalizados por patologías quirúrgicas y que presentan bacteriemias secundarias a procesos sépticos de origen abdominal ${ }^{5,33,36}$. oxacilina fue $57 \%$ y la producción de $\beta$ LEE en los bacilos gramnegativos con porcentajes cercanos a $30 \%$, además de los aislados de $A$. baumannii MDR y $P$. aeuruginosa MDR con porcentajes cercanos a $15 \%$ (Tabla 5).

\section{Discusión}

Teniendo en cuenta las variables analizadas, se observaron algunas similitudes y diferencias con respecto a otros estudios. La utilización de antimicrobianos empíricos previos a la determinación de la bacteriemia se realiza en $88,3 \%$, muy cercano a otros trabajos en Colombia donde su uso alcanza porcentajes cercanos a $86 \% \%^{2,33}$.

Es destacable el tercer lugar que ocupan las infecciones clasificadas con sitio primario de tejidos blandos $(29,7 \%)$ y la gran proporción de hemocultivos positivos, contrariamente a lo descrito en otras series ${ }^{38}$, lo que podría ser debido a la complejidad de nuestros pacientes o a que algunos aislados eran de más de dos sitios anatómicos; esto representa una gran diferencia con respecto a la literatura médica disponible hasta el momento.

La letalidad de nuestros pacientes continúa siendo elevada, próxima a $30 \%$, concordante con la literatura médica disponible, teniendo en cuenta que la mayoría

\begin{tabular}{|c|c|c|}
\hline Variable & OR & IC $95 \%$ \\
\hline $\begin{array}{l}\text { Trombocitopenia después de la toma } \\
\text { de los hemocultivos }\end{array}$ & 26,6 & $3,3-215,4$ \\
\hline Anemia & 17,3 & $2,95-102,0$ \\
\hline $\begin{array}{l}\text { Leucopenia después de la toma de los } \\
\text { hemocultivos }\end{array}$ & 15,6 & $3,6-66,9$ \\
\hline \# 3 hemocultivos positivos & 3,95 & $1,1-13,0$ \\
\hline Índice de PITT grave & 8,8 & $0,8-89,3$ \\
\hline Hipotensión diastólica (PAD $<60$ mmHg) & 4,27 & $0,5-34,9$ \\
\hline Infección polimicrobiana & 3,72 & $0,9-14,4$ \\
\hline Sistema respiratorio & 1,2 & $0,87-1,9$ \\
\hline Sistema vascular & 0,6 & $0,44-0,9$ \\
\hline Edad & 0,45 & $0,14-1,4$ \\
\hline Hospitalización previa & 0,45 & $0,13-1,4$ \\
\hline Piel y tejidos blandos & 0,35 & $0,1-1,1$ \\
\hline Fiebre (temperatura $>38,3^{\circ} \mathrm{C}$ ) & 0,25 & $0,83-0,80$ \\
\hline Infección menor a 48 & 0,17 & $0,2-1,1$ \\
\hline Hipotensión sistólica (PAS < 90 mmHg) & 0,005 & $0,001-0,25$ \\
\hline
\end{tabular}

\begin{tabular}{|c|c|c|c|c|c|}
\hline Antimicrobiano (\% de resistencia) & $\begin{array}{c}\text { Klebsiella } \\
\text { pneumoniae }\end{array}$ & $\begin{array}{l}\text { Escherichia } \\
\text { coli }\end{array}$ & $\begin{array}{c}\text { Acinetobacter } \\
\text { baumannii }\end{array}$ & $\begin{array}{c}\text { Pseudomonas } \\
\text { aeruginosa }\end{array}$ & $\begin{array}{c}\text { Staphylococcus } \\
\text { aureus }\end{array}$ \\
\hline Ceftazidima & 29,6 & 25 & 38,8 & 26,6 & - \\
\hline Cefepime & 19,2 & 9 & 53 & 28,5 & - \\
\hline Cefoxitina & 25,9 & 18,1 & 87,5 & 73,3 & - \\
\hline Aztreonam & 48,1 & 16,6 & 66,6 & 20 & - \\
\hline Ciprofloxacina & 55,5 & 22,2 & 58,8 & 50 & 20 \\
\hline Gentamicina & 50 & 0 & 50 & 22 & 15,3 \\
\hline Meropenem & 16,6 & 12,5 & 46,1 & 30 & - \\
\hline Piperacilina/tazobactam & 33,3 & 14,2 & 50 & 20 & - \\
\hline Cotrimoxazol & 58,8 & 14,2 & 46,6 & 22,2 & 0 \\
\hline Clindamicina & - & - & - & - & 11,7 \\
\hline Oxacilina & - & - & - & - & 50 \\
\hline Vancomicina & - & - & - & - & 0 \\
\hline Rifampicina & - & - & - & - & 0 \\
\hline Linezolid & - & - & - & - & 0 \\
\hline
\end{tabular}


Es llamativo que menos de $20 \%$ de los pacientes en quienes se aísla patógenos de la piel cumplen los criterios de definición de caso y aproximadamente la mitad tiene más de dos muestras positivas lo que impacta en tratamientos antimicrobianos inadecuados, aunque deja ver que para estos patógenos el diagnóstico continúa siendo difícil, por lo cual estos criterios deberían actualizarse.

Es de resaltar el antecedente de la infección por VIH que representa una gran carga de enfermedad en nuestro medio, y ya que Santander es uno de los departamentos de mayor notificación de la enfermedad en el país. Según estudio de Cuervo y cols., esta co-morbilidad sólo se presenta aproximadamente en $4 \%$ de los pacientes según diferentes cohortes analizadas; nuestros datos resaltan la importancia de las infecciones no oportunistas tradicionalmente descritas ${ }^{37}$. Es importante mencionar que nuestros pacientes se presentan con recuentos de LTCD4+ con una media de 29 céls $/ \mathrm{mm}^{3}$, lo que deja ver que el grado de inmunosupresión es el principal factor para desarrollar infecciones graves y que en nuestro hospital se realizan diagnósticos de infecciones avanzadas; más de la mitad corresponden a diagnósticos nuevos ${ }^{38,39}$.

No podemos determinar si el uso de antimicrobianos empíricos está acorde a lo descrito en la literatura científica ya que no era un objetivo del estudio. Sin embargo, este estudio deja ver que los microorganismos MDR son una preocupación creciente, que deben llevar al adecuado uso e implementación de los programas o estrategias con el fin de reducir la letalidad en los pacientes con infecciones asociadas a la atención en salud. Con la tendencia al aumento de la resistencia bacteriana, es necesario fortalecer las políticas institucionales y nacionales del uso racional de antimicrobianos, las estrategias de curación de los pacientes, su seguridad, programas de vigilancia, control, protocolización e intervención en el uso de antimicrobianos (antibiotic stewardship), y el empleo de antimicrobianos que garanticen una respuesta terapéutica equivalente a la de las moléculas innovadoras y con ello, la reducción final de la resistencia ${ }^{4,33,40,41}$.

El análisis multivariado refleja condiciones relacionadas con la letalidad como los estados de sepsis grave y sus complicaciones, entre ellas las más importantes para nuestro estudio (= las hematológicas), la carga bacteriana determinada por la gran proporción de infecciones polimicrobianas y el número de muestras positivas, lo cual no se había reportado previamente según la literatura médica disponible, por lo menos en nuestro medio y en el escenario clínico ${ }^{21,27,33,44,45-50}$.

La gran proporción de pacientes con infección por VIH y su estado inmunológico profundamente comprometido refleja condiciones sociales y de nuestra realidad que no eran objetivo del estudio pero que muestran el probable diagnóstico tardío de la infección en nuestro hospital con impacto en letalidad, aunque sin diferencias a los pacientes sin esta infección teniendo en cuenta el índice de Pitt ${ }^{42,43}$.

Este es el primer estudio en el nororiente colombiano y en la región de Santander con el objetivo de describir las características de los pacientes con patologías médicas que presentan infecciones del torrente sanguíneo. A pesar de que por su carácter retrospectivo y modelo de selección de los pacientes pueden presentarse sesgos, es de resaltar que complementa la información disponible sobre las infecciones del torrente sanguíneo en un escenario diferente a los que se han descrito previamente, con conclusiones importantes desde el punto de vista de resistencia bacteriana y proporción de pacientes con infección por $\mathrm{VIH}^{1-10}$.

\section{Referencias bibliográficas}

1.- Cortés J A, Leal A L, Montañez A M, Buitrago G, Castillo J S, Guzmán L, On behalf of GREBO. Frequency of microorganisms isolated in patients with bacteremia in intensive care units in Colombia and their resistance profiles. Braz J Infect Dis. 2013; 17 (3): 346-52. http:// dx.doi.org/10.1016/j.bjid.2012.10.022.

2.- González A L, Leal A L, Cortés J A, Sánchez R, Barrero L I, Castillo J S, et al. Efecto del tratamiento antibiótico inicial adecuado sobre la mortalidad en pacientes en estado crítico con bacteriemia por Pseudomonas aeruginosa. Biomédica. 2014; 34 (Suppl 1): S58-66. https:// doi.org/10.7705/biomedica.v34i0.1691.

3.- Cortés J A. Garzón D C, Navarrete J A, Contreras K M. Impact of inappropriate antimicrobial therapy on patients with bacteremia in intensive care units and resistance patterns in Latin America. Rev Argent Microbiol. 2010; 42 (3): 230-4. PMID: 21180395.

4.- Kollef M H. Inadequate antimicrobial treatment: an important determinant of outcome for hospitalized patients. Clin Infect Dis. 2000; 31 (Suppl 4): S131-8. doi: 10.1086/314079.

5.- Abraham K, Dolman H S, Zimmerman L H, Faris J, Edelman D A, Baylor A. Impact of inappropriate initial antibiotics in critically ill surgical patients with bacteremia. Am J Surg. 2016; 211 (3): 593-8. doi: 10.1016/j. amjsurg.2015.10.025.

6.- Umemura T, Hamada Y, Yamagishi Y, Suematsu $\mathrm{H}$, Mikamo H. Clinical characteristics associated with mortality of patients with anaerobic bacteremia. Anaerobe. 2016; 39: 45 50. doi: 10.1016/j.anaerobe.2016.02.007.
7.- Christensen J S, Jensen T G, Kolmos H J, Pedersen C, Lassen A. Bacteremia with Streptococcus pneumoniae: sepsis and other risk factors for 30-day mortality: a hospitalbased cohort study. Eur J Clin Microbiol Infect Dis 2012; 31 (10): 2719-25. doi: 10.1007/ s10096-012-1619-5.

8.- García-Vázquez E, Albendín H, Hernández-Torres A, Canteras M, Yagüe G, Ruiz J. Estudio de una cohorte de pacientes con bacteriemias por Enterococcus spp. Factores de riesgo para resistencia de alto nivel a aminoglicósidos. Rev Esp Quimioter. 2013; 26 (3): 203-13. https://pdfs.semanticscholar.org/ d86a/20a844dd0149caf747a5800d3d1d491 bc727.pdf.

9.- Conway L J, Carter E J, Larson E L. Risk factors for nosocomial bacteremia secondary to urinary catheter-associated bacteriuria: A 
systematic review. Urol Nurs. 2015;35(4):191203. PMID: 26402994.

10.- Díaz Granados C A, Zimmer S M, Klein M, Jernigan J A. Comparison of mortality associated with vancomycin-resistant and vancomycin- susceptible enterococcal bloodstream infections: A meta-analysis. Clin Infect Dis. 2005; 41 (3): 327-33. doi: 10.1086/430909.

11.- Mathurin S, Jaimet C, Agüero A, Moro J, del Pino A, Arosio A, et al. Estudio prospectivo observacional de bacteriemia neumocóccica en adultos. Aspectos clínicos y factores pronósticos. Rev Méd Rosario 2008; 74 : 108-21. http://www.circulomedicorosario.org/ Upload/Directos/Revista/fe2ca2Mathurin\%20 et $\% 20$ al.pdf.

12.- Gabastou J M, Agudelo C I, de Cunto M C, Castañeda E, Silva A P, Di Fabio J L. Caracterización de aislamientos invasivos de S. pneumoniae, H. influenzae y N. meningitidis en América Latina y el Caribe: SIREVA II, 2000-2005. Rev Panam Salud Publica. 2008; 24 (1): 1-15. https://www.scielosp.org/article/ $\mathrm{ssm} /$ content/raw/?resource_ssm_path=/media/ assets/rpsp/v24n1/v24n1 a01.pdf.

13.- Anderson D J, Moehring R W, Sloane R, Schmader K E, Weber D J, Fowler V G Jr, et al. Bloodstream infections in community hospitals in the 21st century: A multicenter cohort study. PLoS ONE. 2014; 9 (3): e91713. doi: 10.1371/ journal.pone.0091713.

14.- Molina F J, Díaz C A, Barrera L, De La Rosa G, Dennis R, Dueñas C, et al. Perfil microbiológico de la infecciones en Unidades de Cuidados Intensivos de Colombia (EPISEPSIS Colombia). Med Intensiva. 2011; 35(2): 75-83. http://scielo.isciii.es/pdf/medinte/ v35n2/original2.pdf.

15.- Tumbarello M, Spanu T, Sanguinetti M, Citton R, Montuori E, Leone F, et al. Bloodstream infections caused by extended-spectrum-betalactamase producing Klebsiella pneumoniae: risk factors, molecular epidemiology, and clinical outcome. Antimicrob Agents Chemother. 2006; 50(2): 498-504. doi: 10.1128/ AAC.50.2.498-504.2006.

16.- Adrianzén D, Arbizu A, Ortiz J, Samalvides F. Mortalidad por bacteriemia causada por Escherichia coli y Klebsiella spp. productoras de beta-lactamasas de espectro extendido: cohorte retrospectiva en un hospital de Lima, Perú. Rev Peru Med Exp Salud Publica. 2013; 30 (1): 18-25. http://www.scielo.org.pe/pdf/ rins/v30n1/a04v30n1.pdf.

17.- Park Y S, Bae I K, Kim J, Jeong S H, Hwang $\mathrm{S} \mathrm{S}$, Seo Y H, et al, Risk factors and molecular epidemiology of community-onset extendedspectrum $\beta$-lactamase-producing Escherichia coli bacteremia. Yonsei Med J 2014; 55 (2): 467-75. doi: 10.3349/ymj.2014.55.2.467.

18.- Pitout J D, Laupland K B. Extended-spectrum beta-lactamase-producing Enterobacteriaceae: an emerging public health concern. Lancet Infect Dis 2008; 8 (3): 159-66. doi: 10.1016/ S1473-3099(08)70041-0.

19.- Tuon F F, Kruger M, Terreri M, Penteado-Filho S R, Gortz L. Klebsiella BLEE bacteremiamortality and risk factors. Braz J Infect Dis 2011; 15 (6): 594-8. doi: 10.1590/s141386702011000600016.

20.- Kaya O, Akcam F Z, Gonen I, Unal O, Ceylan T. Risk factors for bacteremia due to extended-spectrum beta-lactamase- producing Escherichia coli in a Turkish hospital. J Infect Dev Ctries, 2013; 7 (7): 507-12. doi: 10.3855/ jidc. 2788 .

21.- Valderrama S L, González P F, Caro M A, Ardila N, Ariza B, Gil F, et al. Factores de riesgo para bacteriemia adquirida en el hospital por Pseudomonas aeruginosa resistente a carbapenémicos en un hospital colombiano. Biomédica. 2016; 36 (Suppl 1): S69-77. http:// www.scielo.org.co/pdf/bio/v36s1/v36s1a10.pdf.

22.- Kim S H, Kwon J C, Choi S M, Lee D G, Park S H, Choi J H, et al. Escherichia coli and Klebsiella pneumoniae bacteremia in patients with neutropenic fever: factors associated with extended-spectrum $\beta$-lactamase production and its impact on outcome. Ann Hematol 2013; 92 (4): 533-41. doi: 10.1007/s00277-012-1631-y.

23.- Hanses F, Spaeth C, Ehrenstein B P, Linde H J, Schőlmerich J, Salzberger B. Risk factors associated with long-term prognosis of patients with Staphylococcus aureus bacteremia. Infection. 2010; 38 (6): 465-70. doi: 10.1007/ s15010-010-0059-3.

24.- Barrero L I, Castillo J S, Leal A L, Sánchez R, Cortés, J A, Álvarez C A, et al. Impacto económico de la resistencia a la meticilina en pacientes con bacteriemia por Staphylococcus aureus en hospitales de Bogotá. Biomédica 2014; 34: 345-53. http://www.scielo.org.co/pdf/ bio/v34n3/v34n3a05.pdf.

25.- Lasa J S, Fernández M L, Finn C, Bruetman J E, Peroni J, Young P. Bacteriemia en pacientes internados con celulitis. MEDICINA (Buenos Aires). 2011; 72(4): 298-304. PMID: 22892081.

26.- Cosgrove S E, Sakoulas G, Perencevich E N, Schwaber M J, Karchmer A W, Carmeli Y. Comparison of mortality associated with methicillin-resistant and methicillin-susceptible Staphylococcus aureus bacteremia: A metaanalysis. Clin Infect Dis 2003; 36 (1): 53-9. doi: 10.1086/345476.

27.- Oliveros A, Uribe N, Sierra P, Jaimes F, González J M. Bacteriemia por enterobacterias resistentes a carbapenems, Un estudio transversal. Infectio 2015; 19 (2): 60-6. http:// www.scielo.org.co/pdf/inf/v19n2/v19n2a03. pdf.

28.- García-Vázquez E, Albendín H, HernándezTorres A, Canteras M, Yagüe G, Ruiz J, et al. Estudio de una cohorte de pacientes con bacteriemias por Enterococcus spp. Factores de riesgo para resistencia de alto nivel a aminoglicósidos. Rev Esp Quimioter 2013; 26 (3): 203-13. PMID: 24080886.

29.- Ng T M, Khong W X, Harris P N A, De P P, Chow A, Tambyah P A, et al. Empiric piperacillin-tazobactam versus carbapenems in the treatment of bacteraemia due to extended- spectrum beta-lactamase-producing Enterobacteriaceae. PLoS ONE. 2016; 11(4): e0153696. doi: 10.1371/journal.pone.0153696.

30.- Hernández M E, Hernández A, Gómez J, Ruíz J, Canteras M, García E. Influencia en la evolución de los pacientes con bacteriemia/ candidemia de la participación activa del especialista clínico en patología infecciosa. Estudio de una cohorte prospectiva. Infectio 2016; 21 (1): 32-8. http://www.scielo.org.co/ pdf/inf/v21n1/0123-9392-inf-21-01-00032.pdf.

31.- Michael G A, Heil L E, Hayes B D. Appropriate antibiotic therapy. Emerg Med Clin North Am 2017; 35 (1): 25-42. doi: 10.1016/j. emc. 2016.08.003.

32.- Organización Mundial de la Salud. Iniciativa mundial en pro de la seguridad del paciente y directrices sobre higiene de las manos en la atención sanitaria. Geneva: WHO; 2005. https:// www.who.int/patientsafety/information_centre/ Spanish_HH_Guidelines.pdf.

33.- Pallares C J, Martínez E. Factores de riesgo asociados a mortalidad en infecciones relacionadas con la atención en salud en un hospital universitario de tercer nivel en Colombia. Biomédica. 2014; 34 (Suppl 1): 14855. http://www.scielo.org.co/pdf/bio/v34s1/ v34s1a17.pdf.

34.- Ye Q F, Zhao J, Wan Q Q, Qiao B B, Zhou J D. Frequency and clinical outcomes of ESKAPE bacteremia in solid organ transplantation and the risk factors for mortality. Transpl Infect Dis 2014; 16 (5): 767-74. doi: 10.1111/tid.12278.

35.- Freifeld A G, Bow E J, Sepkowitz K A, Boeckh $\mathrm{M} J$, Ito J I, Mullen C A, et al. Clinical practice guideline for the use of antimicrobial agents in neutropenic patients with cancer: 2010 update by the Infectious Diseases Society of America. Clin Infect Dis 2011; 52 (4): e56-93. doi: $10.1093 / \mathrm{cid} / \mathrm{ciq} 147$

36.- Castillo J S, Leal A L, Cortés J A, Álvarez C A, Sánchez R, Buitrago G, et al. Mortality among critically ill patients with methicillinresistant Staphylococcus aureus bacteremia: a multicenter cohort study in Colombia. Rev Panam Salud Publica 2012; 32 (5): 343-50. doi: 10.1590/s1020-49892012001100004.

37.- O'Connor J, Vjecha M J, Phillips A N, Angus B, Cooper D, Grinsztejn B, et al. Effect of immediate initiation of antiretroviral therapy on risk of severe bacterial infections in HIVpositive people with CD4 cell counts of more than 500 cells per $\mu \mathrm{L}$ : secondary outcome results from a randomised controlled trial. 
Lancet HIV 2017; 4 (3): e105-12. doi: 10.1016/ S2352-3018(16)30216-8.

38.- Taramasso L, Tatarelli P, Di Biagio A. Bloodstream infections in HIV-infected patients. Virulence 2016; 7 (3): 320-8. doi: 10.1080/21505594.2016.1158359.

39.- Lee C C, Chou Y J, Lin J N, Chu F Y, Tang $\mathrm{H} \mathrm{J}$, Lai C H, et al. Clinical predictors of the leading pathogens in human immunodeficiency virus-infected adults with community-onset bacteremia in the emergency department: The importance of transmission routes. J Microbiol Immunol Infect 2017; 51 (3): 392-400. doi: 10.1016/j.jmii.2016.08.001.

40.- Bauer S, Aubert C E, Richli M, Chuard C. Blood cultures in the evaluation of uncomplicated cellulitis. Eur J Intern Med. 2016; 36: 50-6. doi: 10.1016/j. ejim.2016.07.029.

41.- Dellit H T, Owens C R, McGowan J E Jr, Gerding D N, Weinstein R A, Burke J P, et al. Infectious Diseases Society of America and the Society for Healthcare Epidemiology of America Guidelines for Developing an Institutional Program to Enhance Antimicrobial Stewardship. Clin Infect Dis. 2007; 44 (2): 15977. doi: $10.1086 / 510393$.

42.- Feldman C, Alanee S, Yu V L, Richards G A, Ortqvist A, Rello J, et al. Severity of illness scoring systems in patients with bacteraemic pneumococcal pneumonia: implications for the intensive care unit care. Clin Microbiol Infect 2009; 15 (9): 850-7. doi: 10.1111/j.14690691.2009.02901.x.

43.- Al-Hasan N M, Y J, Bang D W, Yang H J, Baddour L M. External validation of bloodstream infection mortality risk score in a population-based Cohort. Clin Microbiol Infect 2014; 20 (9): 886-91. doi: 10.1111/14690691.12607.

44.- Araoka H, Baba M, Yoneyama A. Risk factors for mortality among patients with Stenotrophomonas maltophilia bacteremia in Tokyo, Japan, 1996-2009. Eur J Clin Microbiol Infect Dis 2010; 29 (5): 605-8. doi: 10.1007/ s10096-010-0882-6.

45.- Kang C I, Chung D R, Ko K S, Peck K R, Song J H, Korean Network for Study of Infectious Diseases. Risk factors for infection and treatment outcome of extended-spectrum $\beta$-lactamase-producing Escherichia coli and Klebsiella pneumoniae bacteremia in patients with hematologic malignancy. Ann Hematol 2012; 91 (1): 115-21. doi: 10.1007/s00277-011$1247-7$.

46.- Rodríguez C N, Rodríguez-Morales A J, García A, Pastran B, Meijomil P. Antimicrobial resistance of Pseudomonas aeruginosa strains isolated from surgical infections in a 7-yearperiod at a general hospital of Venezuela.
Surg Infect (Larchmt) 2006; 7 (3): 269-273. PMID: 16875460.

47.- Rodríguez A J, Niño Cotrina R A, Neyra Pérez C, Rodríguez C N, Barbella R, Lakatos $\mathrm{M}$, et al. Comparative study of antimicrobial resistance of Pseudomonas aeruginosa strains isolated from urinary tract infection in patients from Caracas and Lima. Int $\mathrm{J}$ Antimicrob Agents 2002; 20 (6): 476-7. doi: 10.1093/ $\mathrm{jac} / 47.6 .903$.

48.- Rodríguez A J, Samaniego D R, Soskin A, Rodríguez C N, Canese J, Ortellado de Canese $\mathrm{J}$, et al. Comparative study of antimicrobial resistance of Pseudomonas aeruginosa strains isolated from patients of Caracas and Asunción in a 4-year-period. Chemotherapy 2002; 48(4): 164-7. doi: $10.1159 / 000063867$.

49.- Rodríguez A J, Rodríguez C N, García A, Duque C, Molina N, Barbella R, et al. Antibiotic susceptibility of Enterobacteriaceae species isolated in Venezuela over ten years. $\mathrm{J}$ Chemother 2001; 13 (4): 450-2. doi: 10.1179/ joc.2001.13.4.450.

50.- Rodríguez A J, Nino Cotrina R A, Neyra Pérez C, Rodríguez C N, Barbella R, Lakatos M, et al Comparative study of antimicrobial resistance of Escherichia coli strains isolated from urinary tract infection in patients from Caracas and Lima. J Antimicrob Chemother 2001; 47 (6): 903-4. https://doi.org/10.1159/000063867. 